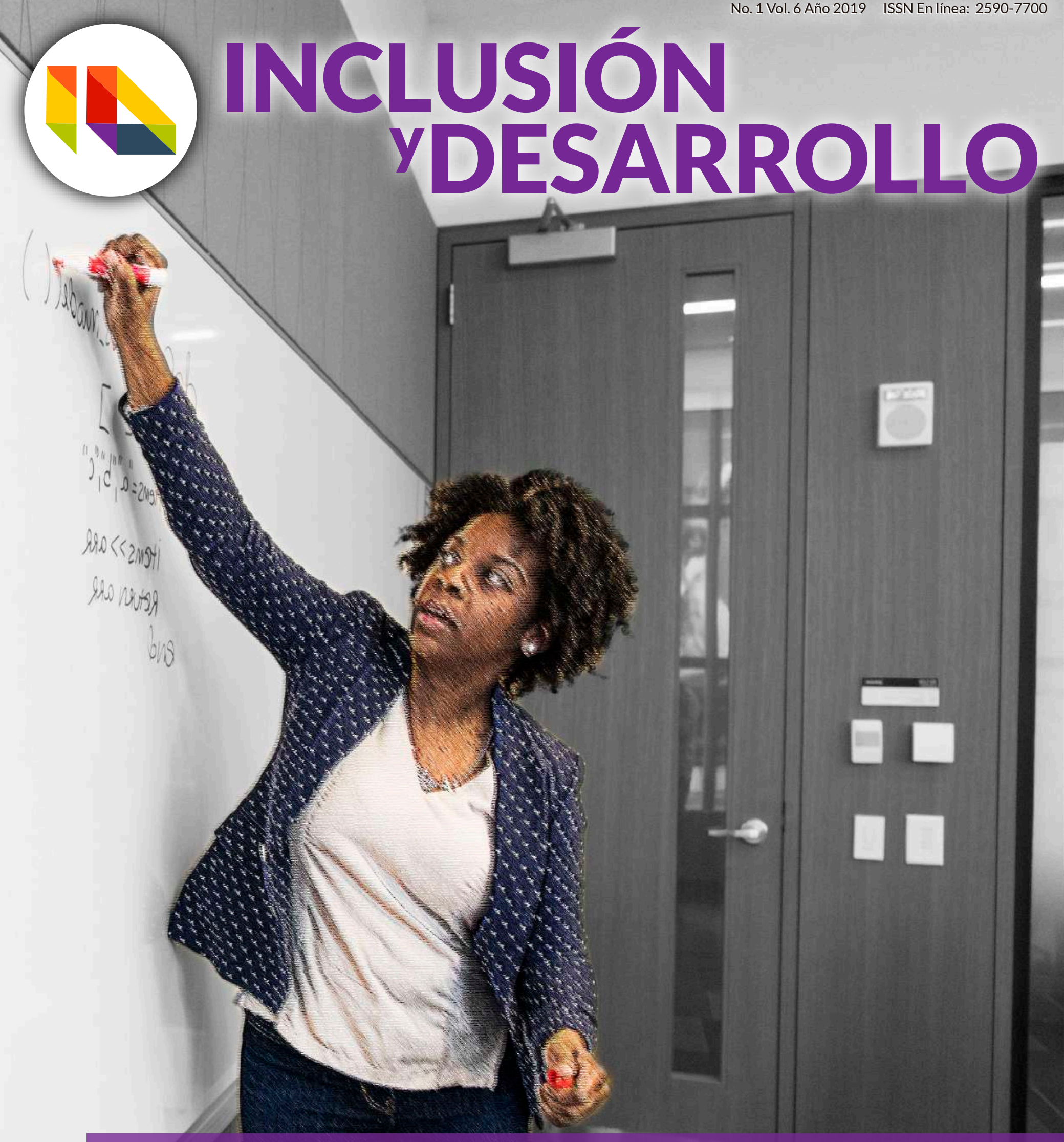

\title{
Relaciones entre el rol del docente y la equidad en educación superior
}


RELACIONES ENTRE EL ROL DEL DOCENTE Y LA EQUIDAD EN EDUCACIÓN SUPERIOR

\section{CONNECTIONS BETWEen TEACHER'S ROLE AND EQUITY IN HIGHER EDUCATION}

\begin{tabular}{lr} 
& $\begin{array}{r}\text { Alexander Restrepo Ramírez } \\
\text { alrestrepora@unal.edu.co }\end{array}$ \\
& $\begin{array}{r}\text { Universidad Nacional de Colombia } \\
\text { Bogotá D.C. - Colombia }\end{array}$ \\
\hline Encuentre este artículo en & Fecha de recepción: 20 de septiembre de 2018 \\
http://revistas.uniminuto.edu/ & Fecha de aceptación 5 de octubre de 2018 \\
Fecha de publicación: 30 de diciembre de 2018
\end{tabular}

\section{Resumen}

Este artículo de revisión presenta una postura crítica y propositiva acerca de la relación entre el rol del docente y el logro de la equidad en educación superior, como una agenda importante en Colombia y la región. Inicia con una aproximación general a los conceptos e indicadores de la equidad en educación superior desde una perspectiva de género y enfoque ético-profesional. Posteriormente, analiza los desafíos del rol del docente en aspectos como las pretensiones de la calidad educativa, el contexto social y la discriminación durante el proceso enseñanza-evaluación. Y finalmente, explora de manera propositiva cómo la perspectiva interseccional podría considerarse una aproximación reflexiva para interiorizar y repensar el rol del docente en la universidad. Se concluye que dadas las circunstancias de la educación superior actual, una práctica pedagógica que no tenga en cuenta la equidad, supone una deficiencia ética.

Palabras clave: equidad, educación superior, rol del docente, práctica pedagógica, ética profesional.

\section{Abstract}

This review article presents a reflection from a critical and proactive approach on the teaching role for a pedagogical practice which contributes to the goal of equity in higher education, as a remarkable agenda in Colombia and Latin America. It begins with an overview to the concepts and indicators of equity in higher education from an ethical-professional approach and gender perspective. Subsequently, it analyzes the challenges of the teacher's role in topics such as the pretensions of educational quality, the social context and discrimination during the teaching-evaluation process. And finally, it explores in a proactive way how the intersectional approach could be considered a reflexive disposition to internalize and reconsider the teacher's role in university. It is concluded that given the circumstances of current higher education, a pedagogical practice that does not take into account equity, implies an ethical deficiency.

Keywords: higher education, teacher's role, pedagogical practice, professional ethics. 


\section{INTRODUCCIÓN}

En los últimos años, principalmente desde la década de los años 90, tanto en Estados Unidos, Europa y Latinoamérica, ha habido una significativa producción académica sobre la equidad y la inclusión en educación superior, debido en parte al reconocimiento del modo en que la diversidad y la desigualdad afectan diversos escenarios sociales (Astin \& Astin, 2015; David, 2009; León y Holguín, 2005). Adicionalmente, en dicho momento se incrementa el debate en torno la formación por competencias (Tobón, 2006), la renovación pedagógica, la evaluación del aprendizaje (Restrepo, 2016) y la ampliación de la cobertura con calidad (Gazzola y Didriksson, 2008).

No obstante, buscar la convergencia entre calidad, cobertura y equidad, ha traído sobre la mesa preocupaciones no sólo por el presupuesto y las políticas más eficientes para lograrla, sino cuestiones acerca del reconocimiento y la justicia distributiva en contextos de diversidad cultural y desigualdades enmarcadas en estructuras sociales racial y étnicamente diferenciadas (Mosquera y León, 2009). Ello remite actualmente a la pregunta por el carácter problemático de la inserción del concepto y la práctica de la equidad en educación superior, ya que si bien tanto en la legislación como en algunas iniciativas institucionales actualmente se pretende un enfoque diferencial y de inclusión (Buelvas et al., 2014; Zapata, Cuenca y Puga, 2014), en la práctica no es del todo claro si la formación de docentes, la planificación curricular o la evaluación del aprendizaje tienen en cuenta el modo en que los marcadores de diferencia ${ }^{1}$ y la formación previa pueden favorecer o no el éxito en la trayectoria académica de todos los estudiantes, y con ello, impactar en un sentido u otro el proceso enseñanza-aprendizaje.

Lo anterior implica que la pregunta por la equidad no depende sólo de los Estados, las instituciones o las medidas de acción afirmativa (Dworkin, 2003, pp. 419-469), sino de la práctica pedagógica, entendida como "La actividad diaria que desarrollamos en las aulas, laboratorios $\mathrm{u}$ otros espacios, orientada por un currículo y que tiene como propósito la formación de nuestros alumnos (...)" (Quero, 2006, p. 90), o bien, como el conjunto de actividades que determinan la función y el papel del docente dentro del contexto educativo (Perrenoud, 2004). De hecho, la práctica pedagógica pareciera no ser un asunto meramente técnico relativo a la aplicación de determinadas "fórmulas" didácticas encaminadas al cumplimiento efectivo del proceso enseñanza-aprendizaje, puesto que:

La pedagogía es, ciertamente, un "saber", pero, ante todo, un "saber hacer" cuyos sujetos son históricos cambiantes, Por eso dice relación con la tecnología, pero no con una ciencia o con unos principios tecnológicos puramente teóricos o inmutables, sino con unos procesos dinámicos, unificados en torno a una intencionalidad. (Molano, 2013, p. 64)

Así las cosas, motiva este trabajo ofrecer algunos criterios para clarificar cuál es la relación entre el rol del docente universitario y el logro de la equidad, asumiendo que tal pretensión es una aspiración abstracta de la justicia mientras no haya un absoluto compromiso junto con una reflexividad sobre la práctica pedagógica aun con sus desafíos y complejidades. Esto no supone que tal favorabilidad a la inclusión y la equidad no existan ya en muchos docentes y en todos los niveles. Más bien indica que independientemente de las políticas o compromisos que adquieran los gobiernos y sus instituciones para promover la equidad en la educación superior, el rol del docente en la cotidianidad es fundamental no sólo por su importancia sino por la incidencia que tiene en los resultados educativos, $\mathrm{y}$ es esto precisamente lo que no se cuestiona suficientemente. Los presupuestos conceptuales y teóricos del análisis surgen como parte de la investigación doctoral denominada Reflexiones sobre la equidad en educación superior en perspectiva interseccional: aproximación a las experiencias de vida de estudiantes de programas de admisión especial en la Universidad Nacional de Colombia, financiada por Colciencias.

Se presentará en primer lugar un abordaje de algunos elementos que desde el plano teórico y práctico suscitan la necesidad de equidad en educación superior como un principio de la jus- 
ticia social y la democracia. En segundo lugar, se ofrecerán algunas ideas relativas a las implicaciones y desafíos del rol docente y la práctica pedagógica dentro de esas demandas de equidad, y finalmente se esbozarán algunas reflexiones para considerar cómo la interseccionalidad podría aportar a una práctica pedagógica reflexiva con la interacción humana, el lenguaje, la evaluación del aprendizaje y el reconocimiento de la diferencia, entre otros aspectos.

\section{¿POR QUÉ HABLAR DE EQUIDAD EN EDUCACIÓN SUPERIOR?}

Se puede entender la equidad como una “(...) idea de igualdad según las necesidades, dándose inicio por esta vía a una transformación importante del rol de la educación el plano de crear una cultura que acepte, respete y asuma las diferencias (aunque sea para llegar a las semejanzas)" (Sleteer, Grant y Padhila, 2011, p. 8). También se ha considerado la necesidad de que todas las personas, independientemente de su condición "racial", étnica o socioeconómica, puedan acceder a instituciones altamente selectivas o de calidad (Astin \& Oseguera, 2004), o que tengan la posibilidad de desarrollar una "voz", esto es, de afirmarse "identitariamente" desde el diálogo horizontal, el debate respetuoso y la movilidad académica (Sellar \& Gale, 2011).

Estas pretensiones se originan en la reconocida desigualdad estructural del sistema educativo, donde es evidente que la formación básica y media no es homogénea en términos de calidad y recursos, lo cual predispone el sistema para que ciertos estudiantes alcancen o no el éxito educativo, y con ello, las oportunidades de movilidad social ${ }^{2}$ (García et al., 2013; Gómez, 2015). Esto es lo que en términos de Bourdieu y Passeron (1996, 2009), determina las posiciones que las personas puedan alcanzar en la sociedad de acuerdo con una reproducción del privilegio social y cultural.

El sistema de educación superior muestra en Colombia que no todos los estudiantes llegan preparados con igual nivel educativo, lo cual puede tener repercusiones en la elección de carrera o institución, y en la capacidad de permanecer en la universidad según los estándares de calidad que actualmente las políticas y normativas plantean como fin transversal. A la vez se observan brechas y asimetrías en cuestiones como el acceso a universidades selectivas y determinadas áreas del conocimiento por género, "raza", etnicidad, condición socioeconómica ${ }^{3}$ o discapacidad (Munévar y Gómez, 2013; Buelvas et al., 2014; Quintero, 2016). En efecto, algunos estudios han identificado que el riesgo de deserción es mayor en estudiantes con menores recursos económicos, ubicándose de manera acumulada para la cohorte del año 2005 en 55,1\% (Sánchez y Márquez, 2012). Actualmente, la deserción por periodo académico o anual se ubica en un 9,0\% en formación universitaria, y en un 17,1\% en formación terciaria (MEN, 2018, p. 21), lo que pone sobre la mesa la conflictividad existente entre las expectativas y los resultados de quienes demandan educación superior en Colombia y otras regiones.

De igual forma, según Cárdenas, Ñopo y Castañeda (2012), en Colombia

Tan sólo 7.4\% de los adultos indígenas asisten a una institución de educación superior, mientras para los afrocolombianos la razón es de uno por cada cinco. Por su parte, 35\% de los jóvenes sin pertenencia étnica asisten a un programa de educación superior, por lo que las brechas entre los grupos sí son considerables. (p. 21)

En el caso de las personas que tienen alguna discapacidad, del 6\% que representan en el total de la población colombiana, $77 \%$ corresponde a los menores niveles de ingresos. $\mathrm{Al}$ mismo tiempo, su nivel educativo se divide en: analfabetismo (22\%); educación básica primaria (46,8\%), y educación superior (5\%), es decir, su tasa de participación universitaria es incluso inferior al de las comunidades indígenas y afrodescendientes (Buelvas et al., 2014, pp. 56-57).

\section{¿QUÉ IMPLICACIONES TIENE EL ROL DEL DOCENTE?}

Aunque las asimetrías podrían considerarse únicamente como el resultado normal de los 
desbalances en la formación previa por regiones, infraestructura y tipo de institución, no es posible ignorar las experiencias de exclusión y discriminación que sufren algunas personas en las universidades. $\mathrm{Al}$ respecto se ha identificado un "racismo cotidiano" como parte de un proceso irreflexivo y "eufemizado" (Quintero, 2014), lo cual, como mostró Fanon (2009), implica una aparente normalización de las exclusiones, las mismas que finalmente son internalizadas por las personas discriminadas bajo la forma de un autodesprecio. En efecto, se ha pretendido minimizar el impacto del racismo argumentando que éste es propio de los sujetos y no de los sistemas, o bien, que sus efectos pueden ser reducidos a partir de un "realismo racial", esto es, la aceptación de las desigualdades como algo consustancial a la jerarquización históricamente establecida (Mosquera y León, 2009).

Ahora bien, la educación implica una posición moral sobre el tipo de cultura que se busca transmitir a la sociedad, de manera que aspectos como la convivencia y el diálogo intersubjetivo son orientados según el tipo de formación recibida (Dewey, 2004). Desde esta perspectiva, para Lipman (1992) la práctica pedagógica debería tener en cuenta que en muchos casos el talento es lo único que tienen los jóvenes de bajos recursos para aspirar a la movilidad social. De lo contrario se estaría condicionando su futuro. No obstante, una visión pesimista al respecto la plantea Bauman (2005) en los siguientes términos: "A decir verdad, el principal propósito de estas escuelas parece ser simplemente la "neutralización" de la juventud considerada despreciable y rebelde, manteniéndola encerrada durante el día, de suerte que, al menos, no se vea implicada en delincuencia callejera”. (p. 109)

Hay buenas razones para no considerar lo anterior meras exageraciones. Un ejemplo es cómo las asimetrías en la educación básica y media representan una desigualdad estructural que afecta las pretensiones de equidad en la educación superior (García et al., 2013), lo cual es cíclico. En efecto, allí también se evidenciará una falta de interés de docentes y directivos sobre cómo lidiar con las desventajas que muchas personas tienen por sus capitales sociales y culturales de origen, lo que se ha mencionado como la "teoría del etiquetaje", donde: "no se pregunta por qué ciertos individuos o grupos se desvían, sino los procesos mediante los cuales las instituciones logran imponer la «etiqueta» de desviados a ciertos grupos - procesos que funcionarán en buena medida como profecía que se autocumple-". (Martín, 1998, p. 30)

Con base en la teoría de la reproducción de Bourdieu y Passeron (1996, 2009), es posible reflexionar sobre la forma en que la estructura social tiende a homogeneizar las prácticas educativas con el fin de privilegiar una sola clase "funcional" e ideal, favorable a los estudiantes que han internalizado dicha cultura dado su capital social, lo cual a menudo se "camufla" en la idea del mérito. Desde el punto de vista de la experiencia y el rol del docente, es comprensible pensar que resulte más sencillo prepararse, actualizarse y ejercer la práctica pedagógica con base en dicha visión unidimensional de los estudiantes, puesto que posiblemente el prestigio tanto del docente como de las mismas instituciones dependa de la eficiencia con que responden a ese único sistema idealmente funcional.

En el caso de la educación superior se ha identificado el modo en que los docentes se adecúan a una práctica pedagógica irreflexiva con los desafíos que plantea la alteridad. En el caso de las identidades de género, Mingo (2006) ha mostrado cómo los comportamientos de algunos docentes en el aula varían dependiendo el sexo de los estudiantes, la popularidad, el liderazgo, el talento e incluso la agresividad que puedan representar:

(...) en la clase de química el número de preguntas formuladas por la docente a los hombres durante el semestre fue marcadamente superior a las que se hicieron a las mujeres (44 y 19) (...); el tipo de preguntas variaba de acuerdo con el sexo: las que implicaban una respuesta con algún tipo de razonamiento eran planteadas a los varones, mientras que las que sólo demandaban información se las hacían a las mujeres. (p. 33)

Respecto a la "raza"/etnicidad, algunos estudios en Colombia han mencionado la idea de un "racismo cotidiano" o eufemístico en la educación superior pública y privada, intentando hacer visible aquello cuya presencia en las relaciones humanas se normaliza o se ignora (Quintero, 2014). Como ejemplo se trae a colación una clase donde se planteó una dinámica entre grupos, justo en la época en que Barack Obama llegó a ser presiden- 
te de Estados Unidos. Finalizada la instrucción de la docente, un estudiante afrodescendiente no quedó en alguna pareja o grupo, mientras los demás estudiantes ya estaban organizados. De pronto alguien dice:

- "Oiga hermano, ¿̇por qué está solo? (...) desde que ganó Obama (...) iquién sabe este tipo qué se está creyendo!”. (Quintero, 2014, p. 83)

Si se observa este hecho de manera minimalista, ello no hace sino parte del conjunto de "bromas" y comentarios despectivos que en torno al género, la orientación sexual, la "raza"/etnia, la clase social o la discapacidad pueden escucharse a diario no sólo en las aulas universitarias sino también en los medios de comunicación y redes sociales. Un ejemplo de ello son los grupos en Facebook de algunas universidades creados por los mismos estudiantes, a menudo bajo el rótulo de "confesiones Uni..." o "Memes Uni..." En estos espacios son comunes las alusiones despectivas hacia las mujeres, los afrodescendientes, los indígenas o las personas con orientación sexual diversa. Podría considerarse que estas situaciones son normales entre pares, o bien, que son propias de los individuos y no de los sistemas. Sin embargo, otros estudios dan cuenta de una discriminación por sexismo o clasismo por parte de los docentes:

Los mismos profesores en clase decían: iváyanse de acá! cuando una preguntaba, [ellos decían] oye las tonteras que preguntai, el profesor entonces decía: tú ¿de qué colegio vení? yo les decía del comercial, iah por eso entonces!, hay harta discriminación sobre todo de los profesores (...) eso es prácticamente es para poder joderte la psique [sic]. (Arancibia et al., 2013, p. 130)

Cuando tales situaciones se normalizan en la cultura universitaria, aunque nadie lo advierta o le dé importancia, no sólo se están afectando vidas particulares, sino que desde lo normativo se estarían violando algunos derechos (ONU, 1948, Arts. 1, 2, 7, 23; ONU, 1966, Arts. 3, Num. 1; 20, Num. 2; 26; Constitución Política de Colombia, 1991, Arts. 5, 13). Sin embargo, la transgresión no está dada únicamente por las violaciones tipificadas en las concepciones universales de la justicia y la equidad, sino por un falso reconocimiento que "(...) no sólo muestra una falta del respeto debido. Puede infligir una herida dolorosa, que causa a sus víctimas un mutilador odio a sí mismas". (Taylor, 1993, pp. 44-45)

Desde el punto de vista del rol del docente, el manejo de este tipo de situaciones no es fácil. Podría decirse que representa un desafío tanto técnico como ético. En lo técnico, la necesidad de responder cada vez más al propósito de la inclusión educativa, entendida "(...) como una versión ampliada de la "integración”, considerando las formas de adaptación de los indivi`duos al sistema; pero también las formas de adaptación de un sistema que tome en cuenta las diversidades de individuos y actores" (Velezmoro et al., 2013, pp. 197-198), implica preguntarse si ello constituye realmente una preocupación para los modelos curriculares de las licenciaturas, la educación continuada o los posgrados destinados a preparación docente. En efecto, es posible que además de la pregunta por los aspectos pedagógicos tendientes a direccionar un proceso de enseñanza-aprendizaje "exitoso", las demandas de equidad supongan nuevas preguntas sobre lo "problémico", lo temático y lo metodológico. En lo ético, la actitud que los docentes pueden tener ante las discriminaciones múltiples implica una toma de posición situada desde la propia experiencia, puesto que posiblemente en algunos casos sean ellos mismos los agentes opresores o las víctimas de discriminación. Para ilustrar esta complejidad y sus correspondientes desafíos, obsérvese el papel que jugó la docente en la situación descrita anteriormente:

Inmediatamente después de que el compañero hubiera mentado el chascarrillo sobre Obama, la profesora se percató de lo incómodo que había podido ser ese comentario para el estudiante afrocolombiano, entonces se acercó a él y le dijo, en un tono algo compasivo y en búsqueda de empatía: "sí, es que el negro está de moda, ¿cierto?”. El estudiante sólo dirigió su mirada hacia ella con una expresión seria y la profesora balbuceó una que otra palabra, como tratando de explicarse a sí misma, pero sin mucho éxito. Finalmente, con una sonrisa que denotaba algo de vergüenza, siguió caminando y se dirigió al fondo del salón, a la espera que todos los estudiantes conformaran parejas para poder iniciar el examen. (Quintero, 2014, p. 84)

Esta descripción (si bien no puede recrear del todo la escena) permite reflexionar sobre 
el modo en que tanto la docente, el estudiante afrodescendiente y los demás compañeros la experimentaron. En el caso de la docente y quien hizo el comentario, podría en principio asumirse que obraron de buena fe; que su intención no fue desarrollar un "falso reconocimiento", ni mucho menos "infligir una herida grave" a la dignidad de una persona. Lo único que ciertamente sugiere el hecho es la dificultad de llevar a cabo una práctica pedagógica que trasciende el deber ser de la enseñanza, puesto que ello suscita cuestionarse constantemente el hacer y el ser de los docentes, así como el ethos universitario en su conjunto. Ante esto, recientemente se ha propuesto la perspectiva interseccional como una manera de reflexionar sobre los desafíos de un contexto universitario cada vez más diverso y a la vez expectante de relaciones equitativas, junto con los desafíos pedagógicos que ello reclama (Munévar y Gómez, 2013; Zapata et al., 2014; Platero, 2014).

La referencia a tales enfoques se justifica en que el problema va más allá de una simple dialéctica entre la formación previa, los espacios o los contextos sociales y culturales en que se presenta la práctica pedagógica. En efecto, un estudio sobre las percepciones de los docentes en torno a la diversidad mostró que: "Desde la "Educación Especial”, el modelo segregacionista considera la diversidad como un déficit y como un problema cuya respuesta se traduce en procesos selectivos y segregadores" (Moliner y Moliner, 2010, p. 24). Resultados similares muestra un estudio donde el $76 \%$ de los docentes universitarios consultados tenían una opinión desfavorable al acceso de estudiantes con alguna discapacidad (Castellanos, Gutiérrez y Castañeda, 2018, p. 166).

Se ha sugerido entonces que la concepción homogénea del estudiantado resulta más conveniente para un único sistema funcional (Sleteer et al., 2011), suprimiendo así la necesidad de repensar los mecanismos de admisión, la diversificación del currículo, los métodos de evaluación del aprendizaje, la capacitación docente e incluso la filosofía institucional, con toda la carga ética que ello demanda (Caldwell, Shapiro y Gross, 2007). De hecho, se ha metaforizado la presencia de indígenas y afrodescendientes en la educación superior como "nuevos sujetos en viejas estructuras" (Castillo y Caicedo, 2008), lo cual se explica en tanto las instituciones podrían considerar que si un estudiante logra acceder, significa a priori que tiene el "mérito" y por ende, puede participar en los espacios académicos en igualdad de condiciones, es decir, igualdad de agencia, competencias y predisposición a las dimensiones epistemológicas preestablecidas. Este prejuicio remite a la idea extendida de una "igualdad formal" que permite a todos los individuos experimentar la equidad bajo el mero estar ahí para competir con sus "pares" por el éxito académico, y por extensión, por el reconocimiento y la movilidad social.

En una investigación sobre las acciones afirmativas en Colombia se documentó la opinión de un docente que frente a la presencia de estudiantes indígenas, afirmó que si bien ellos se reafirman en la defensa del multiculturalismo, a la vez se cobijan en esa misma condición para recibir "prerrogativas académicas" (León y Holguín, 2005, p. 151). En efecto, desde una concepción de la equidad como la planteada ya hace muchos años por Adams (1963), es posible que algunos docentes se sientan tentados a considerar que lo ético es evaluar de manera homogénea y en esa medida, valorar el grado en que los resultados son adecuados o no a los estándares de calidad. La equidad estaría entonces concebida como la operación donde a iguales méritos y esfuerzos, se obtienen los mismos resultados. De hecho, es muy posible que tal concepción estándar de la evaluación del aprendizaje vaya en la dirección del trinomio enseñanza-calidad-competencias, cada vez más extendida en el ámbito universitario (Restrepo, 2016). Sin embargo, una visión crítica de esta actitud ha sido manifiesta desde enfoques que buscan comprender las implicaciones de la diversidad en la educación superior:

Los profesores universitarios ignoran por completo que los conocimientos no pueden ser transmitidos de cualquier forma, ni siguiera los más técnicos, y se resisten a aceptar que los estudiantes puedan estar más interesados en la vida que en sus clases. Recuerdo un caso extremo de infantilismo elevado a la palestra, cuando (...) Se me acercó otro docente aún más joven, acabado de incorporar al equipo, se sentó sobre mi mesa de trabajo como si los papeles ahí depositados fueran invisibles, y me preguntó cómo castigaba a mis alumnos. (Fericgla, 2008, p. 175)

Es posible que las actitudes de algunos docentes dependan de la concepción que tienen de la universidad y de la educación en general, puesto 
que si ésta sólo tiene la finalidad de aportar trabajadores calificados a la sociedad, hablaríamos de una "educación bancaria" que no pretende formar una ciudadanía crítica y solidaria, sino sujetos pasivos afines a los estereotipos del poder y las modas culturales (Zuleta, 2009). En parte, esta fue una de las razones por las que Veblen (1994) defendió el papel de la universidad en la sociedad moderna como una institución cuyo "saber superior" no debería ceder ante las presiones de una cada vez mayor tendencia al lucro y la mercantilización, o a lo que se ha denominado "capitalismo académico" (David, 2009, p. 5). En efecto, si la educación superior suscita en principio una oportunidad de movilidad social y reconocimiento cultural e "identitario" de los diferentes grupos sociales, las demandas de equidad no podrían ser negadas bajo alguna pretensión economicista o instrumental de la universidad (Gazzola y Didriksson, 2008, p. 95).

Sin embargo, estas buenas intenciones chocan a menudo con estructuras curriculares reaccionarias y conservadoras, lo cual supone una presión sobre el rol del docente, su identidad, su ética profesional y su práctica reflexiva:

Por su parte, en el ámbito de la diversidad cultural, el asimilacionismo acepta en los centros a todo el alumnado pero no admite la diversidad de las minorías tratando de suprimirlas y eliminarlas. La cultura dominante absorbe a los diferentes grupos étnicos que conviven en una misma sociedad, y se impone sobre ellos. Desde esta perspectiva el factor de la diversidad se concibe como un problema y una amenaza para el grupo cultural dominante (...). (Moliner y Moliner, 2010, p. 25)

Cuando en la educación superior se da por sentado que sólo ciertas voces, cosmovisiones y competencias pueden legitimar un sistema de méritos y privilegios, se perpetúa la desigualdad, y por ende, la asignación inequitativa de los recursos para becas, pasantías, proyectos de investigación y todo tipo de movilidad académica, puesto que previamente queda asegurado quiénes cumplen los requisitos para obtener dichos resultados (David, 2009). Es ello lo que remite a una visión organicista de la educación, donde pareciera que al docente no le compete ninguna responsabilidad allende su práctica pedagógica con calidad y eficiencia, similar a como en ciertas concepciones realistas y positivistas del derecho se ha pretendido que al juez únicamente le corresponde aplicar la ley:

(...) el grupo de educadores, cuya función consiste en la comunicación de la herencia social, de la experiencia acumulada por toda la especie humana, comunicación que hace posible la "capitalización de la experiencia y los ideales de individuo a individuo, de grupo a grupo, de generación en generación". (De Azevedo, 2015, pp. 137-138)

La complejidad de esta idea radica en que esa misma transmisión de sentidos en torno a la cultura, depende de aquellos códigos y concepciones del mundo que la sociedad acepta o promueve, de manera que la experiencia de exclusión vivida por algunas personas es un reflejo de cómo se ha considerado apto y deseable sólo aquello que según la "cultura hegemónica" puede hacer parte activa de los ideales intelectuales y económicos asimilados. En este sentido, una alta heterogeneidad de instituciones, programas y capacidades académicas conlleva que el desarrollo de la práctica pedagógica no sea la misma en todos los contextos educativos. En este sentido, se afirma que el desempeño de la profesión docente no es ajeno a los factores estructurales, en los cuales media la institución, su prestigio, así como las características sociales, culturales y económicas de la población hacia la cual se dirige (Cataño, 1984, p. 42).

Desde esta perspectiva, no podría generalizarse que el rol del docente tiene a priori una responsabilidad ética sobre el logro de la equidad, puesto que la predisposición ideológica y teleológica depende del contexto socioeconómico y simbólico en el cual ha sido formado y se ubica laboralmente. En efecto, muchos docentes universitarios han sido educados en una cultura académica prestigiosa, por lo cual sería normal que encarnen, defiendan y transmitan sus valores e intereses. Ello daría una apariencia de objetividad sobre su ethos profesional en tanto no tienen otra responsabilidad social más allá del cumplimiento de los estándares de calidad, excelencia y pertinencia en su proceso de enseñanza-evaluación. Sin embargo, cuando se abordan las implicaciones de la equidad en educación superior desde el rol del docente, no es descabellado asumir que su práctica pedagógica puede ser a la vez reflexiva, considerando cómo la diversidad implica una desigualdad traducida 
en capitales culturales, saberes, conocimientos, competencias y dominios lingüísticos que no son del resorte de todas las personas y grupos sociales.

No obstante, es importante recordar que la Ley 30 de educación superior en Colombia estableció la libertad de cátedra y la autonomía universitaria (capítulo VI, artículo $4^{\circ}, 30-32$ ). Estos principios, en el marco de la heterogeneidad de la oferta educativa, también implican el derecho a establecer concepciones particulares de enseñanza-aprendizaje, de manera que cabe preguntarse si es realmente la equidad una preocupación general para el sistema de educación superior, y en esa medida: ¿qué es exactamente aquello que puede aportar el docente en el logro de la equidad educativa?

\section{Algunas consideraciones EN TORNO A UNA PRÁCTICA PEDAGÓGICA PARA LA EQUIDAD}

Independientemente de las variadas perspectivas sobre la relación universidad-sociedad, en la actualidad son fundamentales las preguntas por la no discriminación, el reconocimiento y los espacios de participación para el desarrollo de agencias según múltiples subjetividades. Si bien es problemático considerar que todas las instituciones y docentes tienen una responsabilidad moral con el ideal de la justicia social en la educación superior, es momento de abordar cómo el rol del docente y su práctica pedagógica podrían aportar a este objetivo, considerando tal acción como una realización de la ética profesional reflexiva.

¿Cómo nivelar el campo de juego? Esta pregunta cobra sentido en la metáfora de la educación y la sociedad como canchas de juego en desnivel, donde a priori unas personas tienen más opciones que otras de cumplir las metas (goals) (García et al., 2013). Así, ¿́puede el rol del docente contribuir a balancear tal asimetría? En este punto la perspectiva interseccional podría resultar una herramienta útil no sólo como teoría social sino como metodología para pensar las desigualdades sociales en la educación y otros ámbitos (Platero, 2014). Por interseccionalidad se entiende el modo en que la experiencia de subordinación o privilegio social varía en las personas en razón del género, la orientación sexual, la "raza"/etnia o la clase social, entre otros marcadores de diferencia o sistemas de opresión (Collins, 2015). Así pues, la perspectiva interseccional implica un enfoque a partir del cual tanto los investigadores como los actores sociales pueden ubicarse para comprender las desigualdades y la equidad desde una visión no sólo diferencial sino situada, integral y comprehensiva de la complejidad humana.

Entre los años 2012-2014 Colombia participó en el proyecto MISEAL ${ }^{4}$, del cual surgieron varias investigaciones, congresos y publicaciones con la coordinación del Instituto de Estudios Latinoamericanos de la Universidad Libre de Berlín, Alemania (Zapata et al., 2014). El fin principal de este proyecto fue comprender cómo integrar la perspectiva interseccional para adoptar medidas de inclusión social y equidad en la universidad, teniendo en cuenta las experiencias e imaginarios de estudiantes, docentes y directivos. Uno de los objetivos de esta propuesta fue sensibilizar a todos los actores educativos sobre el modo en que la identidad implica modos distintos de experimentar la opresión por sexismo, clasismo, racismo o cualquier otra actitud social, lo cual podría ayudar a develar aquellas exclusiones que se ignoran, y visibilizar sutilezas al interior de las relaciones humanas que terminan generando malestar, indignación o rechazo por su violencia material y simbólica (Munévar y Gómez, 2013).

Algunas situaciones como la masificación de la oferta educativa, su heterogeneidad y las consecuentes estratificaciones en el acceso a la educación superior de calidad, son a su vez componentes causales de la deserción, usualmente analizada desde sus implicaciones económicas y sociales para el logro de la equidad social (Sánchez y Márquez, 2012, p. 3). No obstante, como se ha reconocido en otros estudios (Buquet, 2013, p. 179), la deserción también afecta la imagen que las personas tienen de sí mismas dentro de una estructura social, y en esa medida, está relacionada con los sentimientos, emociones y "representaciones sociales en torno a la equidad" que van desarrollando los estudiantes. De esta manera, el rol de los docentes es clave en tanto constituyen un poder simbólico que puede generar agencias, inclusión o exclusión en los estudiantes (Arancibia et al., 2013, pp. 130-131).

Con base en estos presupuestos, MISEAL consiguió desarrollar varios enfoques, uno de 
los cuales fue el trabajo de transversalización con estudiantes y docentes (Munévar y Gómez, 2013), con el fin de mostrar que las experiencias situadas de unos y otros implica establecer mecanismos de aprendizaje y diálogo en torno a aquellas situaciones problemáticas que influyen en la adaptación, permanencia y trayectoria académica de personas con múltiples identidades. Esto implica reconocer algo que puede ser incómodo pero sucede, y es el modo en que los docentes, si bien pueden ser agentes opresores, a la vez pueden ser susceptibles de discriminación en uno u otro sentido. Reconocer esto invita a develar que la práctica pedagógica no está al margen de la identidad, mucho menos cuando la autoimagen y los estereotipos construidos socialmente afectan la manera en que las personas se adaptan o no a procesos concretos como la enseñanza, la comunicación oral y escrita (incluyendo idiomas foráneos), el uso de herramientas tecnológicas, la evaluación del aprendizaje y las relaciones interpersonales.

A la luz de lo anterior, ¿̇se puede ser buen docente sin un enfoque de equidad? La respuesta es afirmativa si se parte de la anterior alusión a cómo las modulaciones de la calidad, e incluso la misma idea de justicia, dependen de la formación previa de los docentes, el contexto en el que ejercen su profesión y los imaginarios internos y externos que influyen su práctica pedagógica. De igual forma, desde una perspectiva técnica, se puede ser bueno sin ser justo en tanto "bueno" no sería entonces un valor moral sino una cualidad profesional que remite a la excelencia. La pregunta entonces es si los docentes pueden ser buenos y justos a la vez en su práctica pedagógica, y es ahí en donde la perspectiva interseccional cobra sentido, puesto que dota de significado la cuestión, independientemente de la respuesta dada desde una u otra postura.

Al margen de otras posibilidades analíticas como las pedagogías populares, las pedagogías críticas, la decolonialidad, la etnoeducación, el multiculturalismo e incluso algunos enfoques feministas no acuñados necesariamente bajo este paradigma, la perspectiva interseccional puede generar diferencias en el modo en que se concibe y se experimenta la práctica pedagógica e investigativa, afirmación para la cual es legítimo basarse en la propia experiencia. En efecto, el paso por una variedad de instituciones y niveles educativos como estudiante y docente permite comprender las razones por las cuales se es muy susceptible a observar la equidad desde un enfoque psicologista como el de Adams (1963). Por ende, la ética profesional podría radicar en la coherencia de la gestión educativa con los reglamentos estudiantiles o los sistemas normativos de evaluación del aprendizaje, y en virtud de tal ecuanimidad, lo no ético sería cuestionar a un docente por el fracaso de sus estudiantes, excepto que alguien solicite aclaración de sus calificaciones por considerarlas injustas en ausencia de alguna información más allá del juicio subjetivo y la valoración cuantitativa (Restrepo, 2016). Desde esta perspectiva, pareciera que el sistema universitario funciona bajo la ambivalencia de dos órdenes: el de los fines institucionales (formales) y el de los modos en que se da realmente la práctica pedagógica (ideales-teóricos-didácticos-evaluativos). Cuando hay tensión entre estas dos dimensiones es probable que emerjan concepciones particulares de lo bueno y de lo justo, de lo políticamente correcto y de lo sencillamente honesto. De hecho, es posible que no siempre lo bueno corresponda con lo justo ni lo correcto con lo honesto, y es allí en donde nuevamente la libertad de cátedra implica no sólo compromisos teóricos sino éticos en términos de reflexividad.

Así pues, resulta problemático aceptar que el rol del docente se reduce a la coherencia con la valoración del mérito, dado que si todas las personas son iguales ante la ley pero sustantivamente diversas, lo que sucede en las aulas ha de remitir a una ponderación del éxito o el fallo de la generalmente asimilada "igualdad de oportunidades". En este punto cobran sentido las preguntas: ¿qué se privilegia?; ¿qué se pondera a la luz de las diversas situaciones estudiantiles?, en suma: ¿qué ética debe regular la posición profesional respecto de la equidad? (Caldwell et al., 2007). El modo de plantearse y responderse estas cuestiones depende del enfoque, ya que desde una visión normativa de la calidad educativa el mérito se traduce en inteligencia, trabajo, esfuerzo y dedicación, y la ausencia de mérito, en falta de talento y compromiso (fallo). Es por ello que desde la perspectiva interseccional podría iniciarse por reconsiderar ciertas nociones y actitudes (deconstrucción); continuarse con una reflexividad sobre la práctica pedagógica (eticidad), y finalizarse con su transformación (reconstrucción). 
Si bien recientemente se ha analizado cómo la masificación de la oferta y la demanda ha implicado mayor carga docente sin el respectivo reconocimiento y estímulo (Rama, 2009), asimismo, sensatamente pudiera esperarse que una práctica pedagógica podría ser mejorada aunque implique mayor trabajo (Restrepo, 2016). En efecto, la torsión deconstrucción-eticidad-reconstrucción requiere poner inicialmente en tensión la identidad del docente desde varios marcadores de diferencia, lo cual implica una reflexión sobre ¿cuál es su identidad?; ¿cuáles son sus valores?; ¿cómo impactan tales marcadores su estructura moral y cognitiva o sus ideas y prácticas? En segundo lugar, suscita cuestionarse cotidiana y permanentemente ¿cómo se desenvuelve la práctica pedagógica a la luz de tales principios y valores?, y en tercer lugar implica una nueva práctica pedagógica coherente con dicha reflexividad (Freire y Faundez, 2013).

Esto requiere un análisis detallado del currículo con el fin de identificar si aquello que se pretende enseñar y evaluar puede tener un significado para la vida de los estudiantes, y cómo, desde sus diversas alteridades, pueden verse representados o no por tales cosmovisiones, teorías, declaraciones y prácticas. Tal reevaluación no puede hacerse al margen de la identidad del docente, puesto que desde una perspectiva interseccional es necesario poner en tensión el orden categorial (género, "raza", etnia, condición socioeconómica, discapacidad, orientación sexual, edad, religión) con el orden institucional, experiencial e intersubjetivo (Nuñez, 2014). Con ello, las mismas relaciones de poder requieren una reconfiguración toda vez que la violencia simbólica o material sólo puede ejercerse en ausencia de una reflexividad sobre la identidad propia y ajena. Con este fin, la interseccionalidad otorga herramientas teóricas y metodológicas para conceptualizar y comprender las ventajas o desventajas relativas a las múltiples alteridades (Collins, 2015). De igual forma, dicha reflexión implica considerar la evaluación del aprendizaje de una manera formativa y participativa, con el fin de que las estrategias y los instrumentos de recolección de información encarnen las diferentes agencias, tiempos e intereses de los estudiantes (López, 2009, Restrepo, 2016).

Hacer este tipo de modulaciones en la práctica pedagógica sin duda puede resultar azaroso, peligroso e incluso antiético si por ética profesio- nal se entiende simplemente la conformidad con los estándares normativos. Sin embargo, si tales consideraciones se realizan a la luz de una comprensión del contexto social, cultural y económico en el que se ha de desarrollar dignamente la profesión docente, no hay razón para que las instituciones tengan que sacrificar sus estándares de calidad y eficiencia en favor de prácticas más equitativas. Antes bien, la virtud de un sistema de educación superior en una nación en proceso de superar décadas de desigualdad social, violencia y exclusión, debería ser la integración exitosa de ambas dimensiones.

Consecuentemente, es posible que el conjunto de propuestas del proyecto MISEAL u otras iniciativas permita orientar las inquietudes sobre una práctica pedagógica cuya reflexividad encarna un compromiso de la educación superior con la equidad, y por qué no, aproximarse a la perspectiva interseccional como una forma de ética profesional en donde a la vez que se cumple con la calidad educativa, se promueven valores incluyentes, tolerantes y comprehensivos (MEN, 2018).

Para llevar a cabo un programa de esta naturaleza, sería necesario repensar los modos en que algunos docentes permanecen en una zona de confort que es en sí misma irreflexiva frente al contexto consustancial a los orígenes familiares y étnicos de un estudiantado cada vez más diverso. Al mismo tiempo, demanda un rechazo absoluto frente a toda forma de discriminación, violencia simbólica, verbal o física ejercida por y hacia algún miembro de la comunidad educativa. Por último, implica cuestionar los procedimientos hegemónicos a través de los cuales se ha legitimado el conocimiento científico y social, de manera que otras cosmovisiones, prácticas, discursos e intereses puedan hacer parte del corpus epistémico, y en esa medida, tener espacios equitativos de participación en publicaciones, foros, simposios y encuentros académicos. Si institucionalmente esta postura resulta incompatible, es parte también de la libertad de cátedra subvertir un orden que en su mayor parte ha privilegiado al privilegio. La apuesta entonces es por una práctica pedagógica que aporte al cuestionamiento de las lógicas consuetudinarias que han jerarquizado la sociedad desde la educación, y por ende, han contribuido a una reproducción constante de la desigualdad y la exclusión social. 


\section{Conclusiones}

Fue evidente durante este trabajo que a pesar de que en los últimos años ha habido una amplia preocupación por la inclusión y la equidad educativa en Colombia y la región, lo cual ha llevado a una mayor presencia de estudiantes pertenecientes a grupos sociales que antes tenían escaza o nula participación en la universidad, han surgido problemáticas asociadas a la diversificación de la oferta con consecuencias en la calidad educativa, el incremento de la deserción y la difícil adaptación de estudiantes cuya identidad de género, "racial”, étnica o socioeconómica, entre otras, siguen generando asimetrías visibles en su experiencia de vida y trayectoria académica.

Surgió además de lo expuesto que si bien las demandas de equidad han venido de la mano de iniciativas para favorecer el acceso y la permanencia de estudiantes con alguna dificultad para costear y afrontar los estudios universitarios, los análisis principalmente se han enfocado en los factores estructurales de las oportunidades educativas, cuando hay aspectos significativos que remiten a las relaciones entre los actores educativos. Ello indica que el rol del docente y la práctica pedagógica deben ser analizados igual- mente como componentes fundamentales de la equidad educativa.

Así las cosas, el ideal de equidad en educación superior suscita desafíos éticos y profesionales del docente en torno al éxito o fracaso que los estudiantes puedan durante su trayectoria académica en instituciones públicas o privadas, aunque los contextos heterogéneos deberían llevar a investigaciones y análisis que den cuenta de esas diferencias, con el fin de direccionar mejor las respectivas medidas de inclusión y equidad.

Por último, se ha defendido que la perspectiva interseccional puede ser considerada una herramienta para repensar la vida universitaria en términos de las relaciones entre la enseñanza, la calidad y la equidad, siempre evaluando cómo la educación superior se convierte en un bien determinante para la vida de muchas personas, sobre todo de aquellas cuya identidad ha representado alguna desventaja social para el goce de sus derechos. Por ende, el rol del docente debería ser analizado como un pilar en la reconfiguración del papel social de la universidad como espacio de confluencia de las profesiones y el proyecto de vida de los estudiantes, constituyendo una pregunta ética sobre qué hay más allá de la práctica pedagógica misma como acción social 


\section{Referencias bibliográficas}

Adams, J. S. (1963). Toward an Understanding of Inequity. Journal of Abnormal Psychology, 67(5), 422-436. http://doi. org/10.1037/hoo40968

Arancibia, Susana; Rodríguez, Germán; Fritis, Romina; Tenorio, Nitza y Poblete, Héctor (2013). Representaciones sociales en torno a equidad, acceso y adaptación en educación universitaria. Psicoperspectivas, 12(1),116 $\square$ 138.https://scielo. conicyt.cl/scielo.php?pid=So718-

Astin, Alexander W., \& Oseguera, Leticia (2004). The declining “equity” of American higher education. The Review of Higher Education, 27(3), 321-341. http://doi.org/10.1353/rhe.2004.0001

Astin, A. W., \& Astin, H. S. (2015). Achieving Equity in Higher Education: The Unfinished Agenda. Journal of College and Character, 16(2), 65-74. http://doi.org/10.1080/2194587X.2015.1024799

Bauman, Z. (2005). Vidas desperdiciadas. La modernidad y sus parias. Barcelona: Paidós.

Bourdieu, P. y Passeron, Jean-C. (1996). La Reproducción, Elementos para una teoría del sistema de enseñanza. Madrid: Editorial Popular.

Bourdieu, P. (2009). Los herederos. Los estudiantes y la cultura. 2a ed. Buenos Aires: Siglo XXI.

Buquet, A. (2013). ¿Sólo el género importa? una mirada interseccional a los obstáculos que enfrentan las académicas. En M. Zapata; S. García y J. Chan de Ávila (Eds.), La interseccionalidad en debate. Actas del Congreso Internacional "Indicadores Interseccionales y Medidas de Inclusión Social en Instituciones de Educación Superior" (pp. 169-181). Berlín, Alemania: Instituto de Estudios Latinoamericanos/Freie Universität Berlin.

Buelvas, J. I.; Cobos, A.; Gómez, A. Y.; Henao, D. F.; Murillo, Y. C.; Osorio, J. D.; Cerón, K. R. y Rivera, M. L. (2014). Situando y sintiendo experiencias estudiantiles. Aportes para una Universidad más inclusiva y equitativa desde una perspectiva interseccional. Bogotá: Universidad Nacional de Colombia. MISEAL. Instituto de Estudios Latinoamericanos Freie Universität Berlin.

Bula, J. I. (2009). Equidad social en la educación superior en Colombia. En A. Zerda et al. (comps.). La educación superior: tendencias, debates y retos para el siglo XXI: sostenibilidad y financiación, (pp. 281-310). Bogotá: Universidad Nacional de Colombia.

Castillo, E. y Caicedo, J. A. y (2008). Indígenas y Afrodescendientes en la universidad colombiana: Nuevos sujetos, viejas estructuras. Cuadernos Interculturales 6(10), Chile: Universidad de Valparaiso, 62-90. http://www.redalyc.org/ $\mathrm{html} / 552 / 55261005 /$

Caldwell, C., Shapiro, J. P., \& Gross, S. J. (2007). Ethical leadership in higher education admission: Equality vs. equity. Journal of College Admission, (195), 14-19. Retrieved from http://search.ebscohost.com.ezproxy.liv.ac.uk/login.aspx?di rect $=$ true $\& \mathrm{db}=\mathrm{a} 9 \mathrm{~h} \& \mathrm{AN}=24453161 \&$ site $=$ eds-live\&scope $=$ site

Cárdenas, J. C.; Ñopo, H. y Castañeda, J. L. (2012). Equidad en la Diferencia: Políticas para la Movilidad Social de Grupos de Identidad. Misión de Movilidad Social y Equidad (No. 010319). Bogotá: Universidad de los Andes-CEDE.

Castellanos, C; Gutiérrez, A; Castañeda, J. (2018). Actitudes hacia la discapacidad en educación superior. Revista Inclusión y Desarrollo, 5(2) 2018, 157-169. http://biblioteca.uniminuto.edu/ojs/index.php/IYD/article/view/1611

Cataño, Gonzalo (1989). Educación y Estructura Social. Bogotá: Plaza y Janes.

Collins, P. H. (2015). Intersectionality's Definitional Dilemmas. Annual Review of Sociology, 41, 1-20. http://doi. org/10.1146/annurev-soc-073014-112142

David, Miriam E. (2009). Diversity, gender and widening participation in global higher education: a feminist perspective. International Studies in Sociology of Education, 19(1), 1-17. https://doi.org/10.1080/09620210903057590

De Azevedo, F. (2015). Sociología de la educación. México: Fondo de Cultura Económica.

Dewey, John (2004). Democracia y educación: una introducción a una filosofía de la educación (6a ed). Buenos Aires: Losada.

Dworkin, R. (2003). Virtud soberana. La teoría y la práctica de la igualdad. Barcelona, España: Paidós.

Fanon, F. (2009). Piel negra, máscaras blancas. Madrid, España: Akal. 
Fericgla, J. M. (2008). Las experiencias activadoras de estructuras (exaces) en el desarrollo de las sociedades y de los individuos. En C. E. Pinzón; G. Garay, \& R. Suárez (Comps./Eds), Para cartografiar la diversidad de las jóvenes (pp. 143-187). Bogotá: Universidad Nacional de Colombia.

Freire, P., y Faundez, A. (2013). Por una pedagogía de la pregunta. Crítica a una educación basada en respuestas a preguntas inexistentes. Buenos Aires: Siglo XXI.

García, M.; Espinosa, J. R.; Jiménez, F.; Parra, J. D. (2013). Separados y desiguales. Bogotá, Colombia: Dejusticia.

Gazzola, A. L. y Didriksson A. (Eds.) (2008). Tendencias de la educación superior en América Latina y el Caribe, (pp. 2154). Caracas, Venezuela: IESALC-UNESCO.

Gómez, V. (2015). La pirámide de la desigualdad en la educación superior en Colombia. Diversificación y tipología de instituciones. Bogotá: Universidad Nacional de Colombia.

León, M. y Holguín, J. (2005). Acción afirmativa en Colombia. Hacia democracias inclusivas. Santiago, Chile: Fundación EQUITAS.

Lipman, M. (1992). La filosofía en el aula. Madrid, España: Ediciones de la Torre.

López, V. M. (coord.) (2009). Evaluación formativa y compartida en Educación Superior. Propuestas, técnicas, instrumentos y experiencias. Madrid: Narcea.

Martín, E. (1998). Producir la juventud: crítica de la sociología de la juventud. Madrid, España: Akal.

Mingo, A. (2006). ¿Quién mordió la manzana? Sexo, origen social y desempeño en la Universidad. México: Fondo de Cultura Económica.

Ministerio de Educación Nacional -MEN- (2018). Enfoque e identidades de género para los lineamientos política de educación superior inclusiva. Bogotá: Ministerio de Educación Nacional.

Moliner, L. \& Moliner O. (2010). Percepciones del profesorado sobre la diversidad. Estudio de un caso. Revista de educación inclusiva, 3(3), 23-34. https://dialnet.unirioja.es/descarga/articulo/3607985.pdf

Molano, M. (Comp.) (2013). Una universidad Humanística. Bogotá, Colombia: Universidad de La Salle.

Mosquera, C. y León, R. E. (2009). Acciones Afirmativas y ciudadanía diferenciada étnico-racial negra, afrocolombiana, palenquera y raizal. Entre Bicentenarios de las Independencias y Constitución de 1991. (pp. I-XXXVII). Bogotá: Universidad Nacional de Colombia. Bogotá, Colombia: Universidad Nacional de Colombia.

Munévar, D. I. y Gómez, Y. (2013). Rutas posibles desde el sur. Guía para hacer transversalidad y continuar transversalizando la inclusión social y la equidad en la formación y la investigación. Bogotá: Universidad Nacional de Colombia.

Nuñez, Anne-Marie (2014). Advancing an Intersectionality Framework in Higher Education: Power and Latino Postsecondary Opportunity. In M. D. Paulsen (Ed.), Higher Education: Handbook of Theory and Research, 29. https:// link.springer.com/chapter/10.1007/978-94-017-8005-6_2

Organización de Naciones Unidas (1948). Declaración Universal de los Derechos Humanos. Recuperado de http://www. derechoshumanos.net/normativa/normas/1948-DeclaracionUniversal.htm?gclid=CJ-S6oy6_M8CFYJkhgodIPcCIw

Organización de Naciones Unidas (1966). Pacto Internacional de Derechos Civiles y Políticos. Recuperado de http://www. corteconstitucional.gov.co/relatoria/pacto\%20internacional\%20de\%20derechos\%20civiles\%20y\%20politicos.php

Perrenoud, P. (2004). Desarrollar la práctica reflexiva en el oficio de enseñar: profesionalización y razón pedagógica (Vol. 1). Barcelona: Graó.

Platero, R. L. (2014). Metáforas y articulaciones para una pedagogía crítica sobre la interseccionalidad. Quaderns de psicología, 16(1), 55-72. https://www.raco.cat/index.php/QuadernsPsicologia/article/view/292562

Quero, V. D. (2006). Formación docente, práctica pedagógica y saber pedagógico. Laurus, 12(Ext), 88-103. http://www. redalyc.org/html/761/76109906/

Quintero, O. A. (2014). El racismo cotidiano en la universidad colombiana desde la experiencia vivida por los estudiantes negros en Bogotá. Universitas humanística, 77, 71-94. http://www.redalyc.org/html/791/79130107004/

Quintero, O. A. (2016). La creciente exclusión de las mujeres de la Universidad Nacional de Colombia. Nómadas, (44), 123145. http://www.scielo.org.co/scielo.php?pid=S0121-75502016000100008\&script=sci_abstract\&tlng=en 
Rama, C. D. (2009). La tendencia a la masificación de la cobertura de la educación superior en América Latina. Revista iberoamericana de educación, (50), 173-195. https://core.ac.uk/download/pdf/41563563.pdf

República de Colombia. Congreso de la República (2015). Constitución Política de Colombia. Actualizada con los Actos Legislativos a 2015. Bogotá: Corte Constitucional de Colombia, CENDOJ.

Restrepo, A. (2016). El malestar en la evaluación del aprendizaje en educación superior. Horizontes Pedagógicos, 18(1), 138152. http://revistas.iberoamericana.edu.co/index.php/rhpedagogicos/article/view/935

Sánchez, F. y Márquez J. (2012). La deserción en la Educación Superior en Colombia durante la Primera Década del Siglo XXI: ¿'Por qué ha aumentado tanto? Serie Documentos Cede, 2012-31. Bogotá: Universidad de los Andes. http://2013. economicsofeducation.com/user/pdfsesiones/162.pdf

Sellar, S., \& Gale, T. (2011). Mobility, aspiration, voice: a new structure of feeling for student equity in higher education. Critical Studies in Education, 52, 115-134. http://doi.org/10.1080/17508487.2011.572826

Sleteer, Christine; Grant, Carlo; Padhila, Paulo Roberto (2011). Educación Multicultural Práctica de la equidad y diversidad para un mundo que demanda esperanza. Talca, Chile: Ediciones Universidad de La Frontera.

Taylor, C. (1993) Multiculturalismo y la política del reconocimiento. México: Fondo de Cultura Económica.

Tobón, S. (2006). Competencias en educación superior. Políticas hacia la calidad. Bogotá: ECOE.

Veblen, T. (1994). El lugar de la universidad en la vida moderna. Revista Colombiana de Educación, (28), 7-42.

Velezmoro, C.; Chávez, J.; Rodrich, H., y Bacal, A. Equidad e inclusividad en el sistema de educación superior en el Perú (2013). En: O. Espinoza (Ed.). Equidad e inclusividad en la educación superior en los países andinos: los casos de Bolivia, Chile, Colombia y Perú (pp. 197-243). Chile: Ediciones Universidad UCINF-Red Iberoamericana de Investigación en Políticas Educativas.

Zapata, M., Cuenca, A., y Puga, I. (2014). Guía desde un enfoque interseccional. Metodología para el Diseño y Aplicación de Indicadores de Inclusión Social y Equidad en Instituciones de Educación Superior de América Latina. Berlín: Instituto de Estudios Latinoamericanos, Freie Universität Berlin.

Zuleta, E. (2009). Educación y democracia (9a ed.). Bogotá: Fundación Hombre Nuevo Editores. 
Revista incluida en los siguientes agregadores de contenidos
Revista incluida en los siguientes directorios

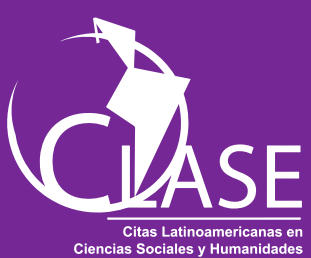

llboblat

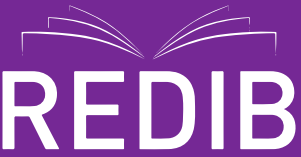

Red Iberoamericana

de Innovación y Conocimiento Cientifico

\section{Google Académico}
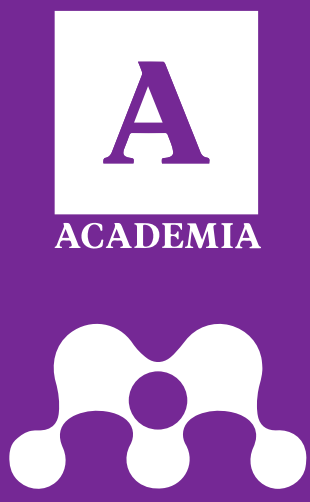

MENDELEY

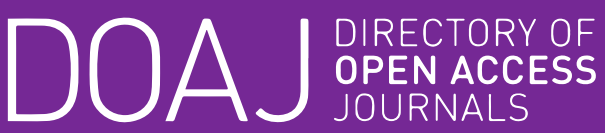

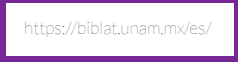
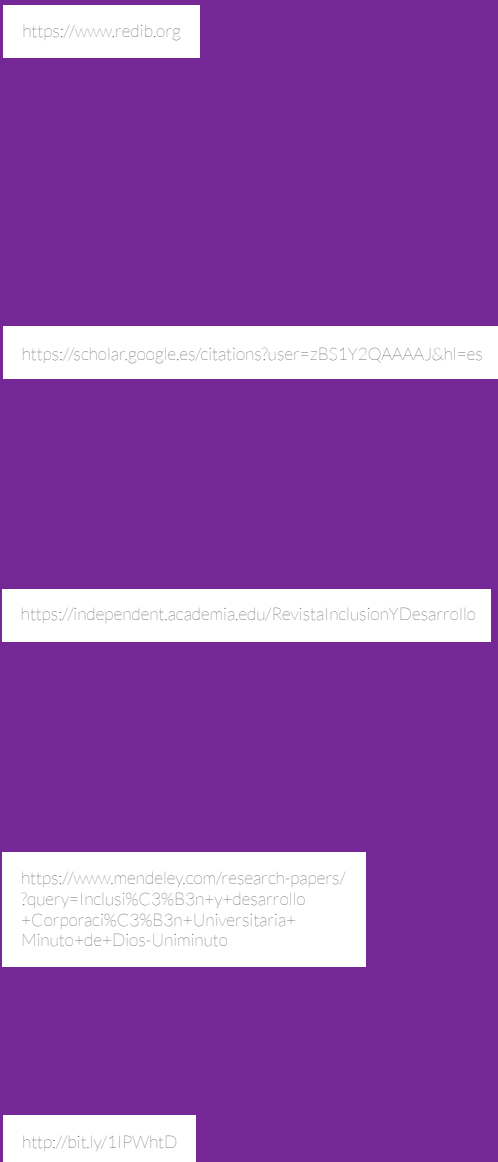

Revista incluida en la siguiente red social

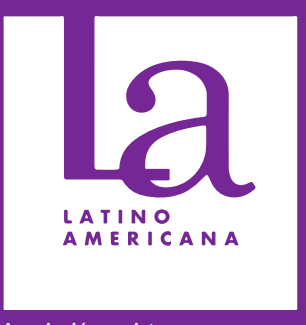



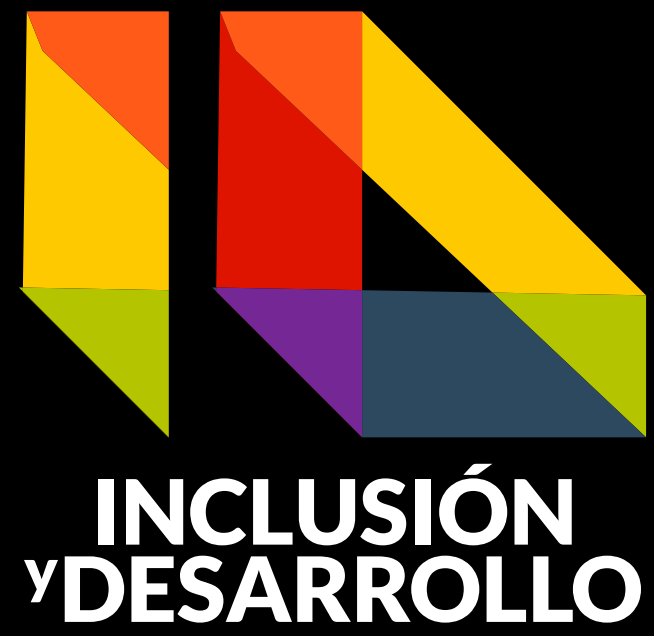

No. 1 Vol. 6 Año 2019 ISSN En línea: 2590-7700

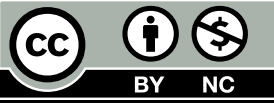

